\title{
Epidemiologia del latrodectismo en la Provincia de Buenos Aires, Argentina
}

\section{Epidemiology of latrodectus bites in Buenos Aires Province, Argentina}

\author{
Carlos S. Grisolia*, Fabio O. Peluso ${ }^{\star \star}$, Nestor O. Stanchi**, Flavio Francini ${ }^{\star \star}$
}

\begin{abstract}
GRISOLIA, C. S. et al. Epidemiologia del latrodectismo en la Provincia de Buenos Aires, Argentina. Rev. Saúde públ., S. Paulo, 26:1 - 5, 1992. El presente trabajo tiene por objeto dar a conecer los resultados del Latrodectismo y su tratamiento especifico, durante un periodo de 10 años (1979-1988) en la Provincia de Buenos Aires. Se suministran datos de la distribución de casos por año, por meses, incidencia según sexo, residencia del accidentado, región corporal de la picadura, sintomatologia presentada, tiempo transcurrido entre el accidente y la aplicación del suero antilatrodectus y captura del animal agresor. Con respecto al año se produjo um pico entre 1982 y 1983 con 38 accidentes, correspondiendo al periodo de 10 años una media de 28,1 accidentes anuales los que presentan mayor incidencia de diciembre a marzo. Con respecto al sexo y residencia del accidentado un $80 \%$ corresponde a hombres, siendo principalmente trabajadores rurales los afectados. El mayor porcentaje de accidentados se produjo en antebrazos, cintura pélvica y muslos. Con respecto al tiempo transcorrido desde el accidente hasta la aplicación del suero, el $46 \%$ lo realiza entre las tres primeras horas. Sólo un $15 \%$ capturó al animal agresor.
\end{abstract}

Descriptores: Aracnidismo, epidemiologia, Latrodectus.

\section{Introducción}

En la provincia de Buenos Aires, y en general en toda la República Argentina, las especies del Género Latrodectus (Aranea: Theriidae) ocasionan accidentes graves en el hombre, a pesar de ello los casos mortales son excepcionales ${ }^{6,8}$. A las especies de este Género se las conoce con los nombres vulgares de "viuda negra", "del lino", "rastrojera", y "tarántula". Latrodectus mirabilis tiene una amplia distribución en la Argentina y es la especie que se encuentra en la Provincia de Buenos Aires. Es posible encontrar ejemplares en toda la provincia a excepción de la zona húmeda del Delta de Paraná y de la costa atlantica. Sin embargo, también se han encontrado ejemplares en estas áreas, comprobándose que las arañas habian sido transportadas en forma accidental en fardos de pasto.

L. mirabilis tiene hábitos rurales, vive al aire libre, a nivel del suelo, debajo de piedras, bolsas de cereales, dentro de

* Dirección Provincial de Medicina Sanitaria. Ministerio de Salud de la Provincia de Buenos Aines - Argentina.

** Laboratorio y Museo de Animales Venenosos (LYMAV). Facultad de Ciencias Médicas. Universidad Nacional de La Plata.

Separatas/Reprints: C.S. Grisolia. Calle 51 n. $1120-1900$

- La Plata - Argentina osamenta seca, entre hierbas, en el rastrojo, plantaciones de ajo, papas, etc. También en galpones, depósitos y viviendas rurales. Es poco agresiva, sólo reacciona si se la molesta o cuando accidentalmente se la comprime sobre alguna región corporal ${ }^{1,2}$.

Con el objeto de brindar atención sanitaria rápida y eficiente de los accidentes humanos provocados por animales venenosos, se crearon en la Provincia de Buenos Aires, 22 Centros Antiponzoñosos en zonas donde los registros de captura demostraron la presencia de ofidios $y / 0$ arañas de interés toxicológico ${ }^{3}$.

El presente trabajo tiene la finalidad de dar a conocer los resultados del latrodectismo y su tratamiento especifico, durante um periodo de 10 años (1979-1988) en la Provincia de Buenos Aires.

\section{Material y Metodos}

Cada Centro Antiponzoñoso recibe como depositario una cantidad de suero antilatrodectus, éste es presentado en ampollas de $2 \mathrm{ml}, \mathrm{y}$, de acuerdo a las indicaciones terapéuticas del Laboratorio Productor, una sola ampolla es suficiente como dosis única y total. Para la reposición del suero utilizado, se lo solicita mediante um Formulario 
Epidemiologico (FE), debidamente cumplimentado en todos sus rubros por el médico actuante. Debido a la poca disposición del equipo de salud en llenar formularios extensos, se diagramó um FE solamente con los datos de mayor utilidad para el control del suero especifico. Este FE brinda a las autoridades sanitarias los datos del Latrodectismo en el ámbito provincial.

Durante el periodo de observación (1979-1988), se evaluó la distribución de los accdidentes por año, por meses, incidencia según sexo, residencia del accidentado, región corporal del sitio de la picadura, sintomatologia presentada, tiempo transcurrido entre el accidente y la aplicación del suero antilatrodectus y si hubo captura del animal agresor.

\section{Resultados y Discusión}

En la evaluación de los casos de araneismos tratados con suero antilatrodectus, atendidos por los Centros Antionzoñosos durante el periodo de 10 años, se notificaron 281 accidentes sin registrar casos mortales.

En la Figura 1 puede observarse, discriminados año por año, la cantidad de accidentes producidos en ese periodo. Al descenso casuistico a partir del año 1984, no se encuentra una causa predominante. Entre las variables que pudieran haber influido se destaca la falta de continuidad de las normas de notificación de los casos, provocadas por cambios en el personal administrativo y

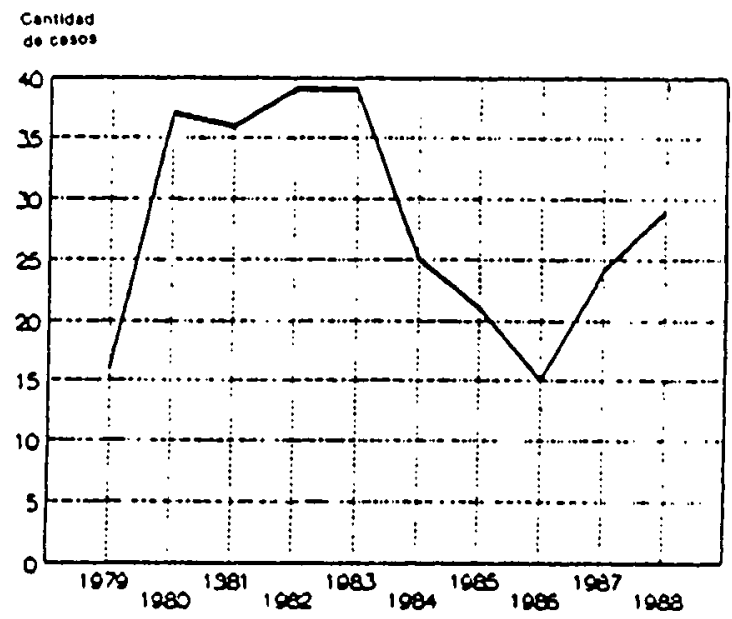

Figura 1. Cantidad de accidentes por año, tratados con suero antilatrodectus, Provincia de Buenos Aires. 1979.1988 .

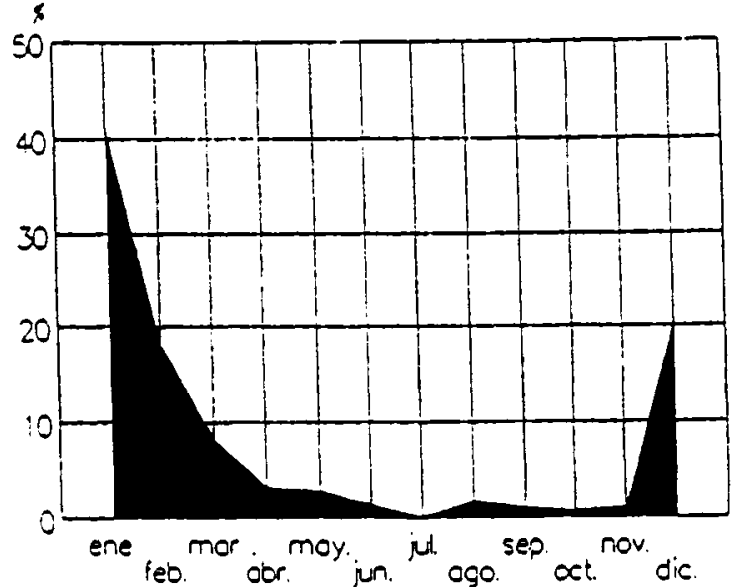

Figura 2. Incidencia porcentual de accidentes tratados con suero antilatrodectus, segun meses del año, Provincia de Buenos Aires. 1979-1988.

técnico de la mayoria de los establecimientos hospitalarios provinciales.

En la Figura 2 se puede apreciar la distribución porcentual de accdidentes provocados por arañas de acuerdo a los meses del año; el mismo muestra el mayor porcentaje de diciembre a marzo. Se observa un marcado descenso en abril-mayo presentandose algunos casos aislados hasta el mes de noviembre; si bien estos últimos recibieron tratamiento a base suero antilatrodectus, se duda que la araña causante haya sido Latrodectus, ya que ésta tiene su actividad entre los meses de diciembre a marzo, época factible de latrodectismo en la Provincia de Buenos Aires. Al respecto, trabalhos sobre bioecologia de esta especie ${ }^{7}$, demuestran claramente la ausencia de ejemplares adultos en el periodo invernal, estudio que sustenta nuestras dudas sobre la especie causante de estos accidentes. Asimismo es interesante señalar la diferencia que existe con respecto a la Provincia de Santiago del Estero (Norte de Argentina), donde los meses de marzo, abril y mayo registran el pico máximo de latrodectismo ${ }^{12}$.

Con respecto a la incidencia según sexo de la persona afectada, el $80 \%$ de los accidentes se producen en el hombre. La coincidencia entre la actividad máxima de esta especie, el lavantamiento "a mano" de algunas cosechas (ajo, cebolla, papas) hace que el hombre, por sus tareas rurales, sea el más expuesto. En este análisis, tanto la causa del accidente, como la incidencia del sexo, 


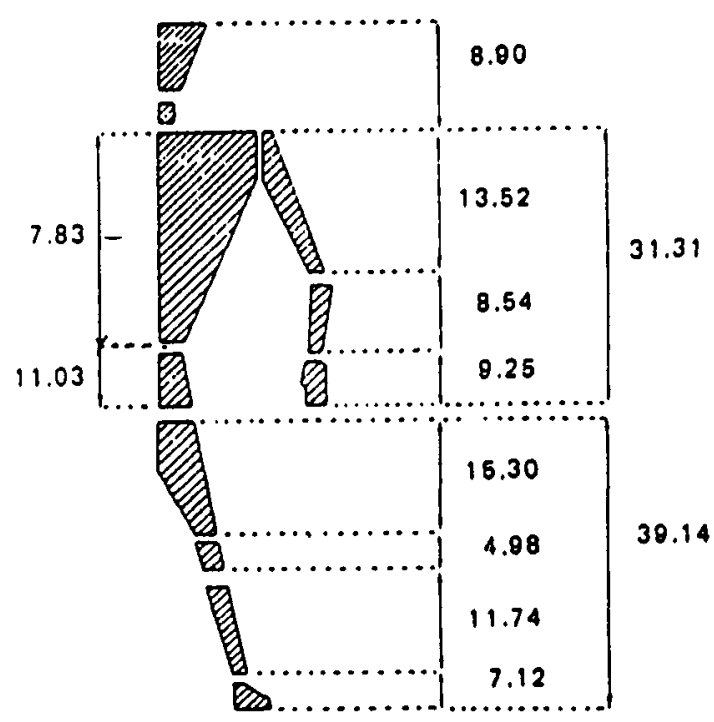

Figura 3. Topograma porcentual del lugar afectado por accidentes tratados con suero antilatrodectus, Provincia de Buenos Aires. 1979-1988.

son totalmente concordantes con estudios similares ${ }^{10}$.

Los residentes en zohas donde abunda L. mirabilis y que desarrollan actividad rural, están más expuestos, ya que del total de casos, el $94 \%$ corresponde a éstos.

En la Figura 3 se observa un topograma porcentual del lugar del cuerpo afectado. Puede apreciarse que la distribución es levemente superior en antebrazos, cintura pélvica y muslos. El pequeño tamaño de la araña y la abundancia en que se encuentra en determinadas áreas, hace que no puedan evitarse los accidentes ne cualquier parte del cuerpo.

Tabla 1. Incidencia de la sintomatologia presentada por accidentes tratados con suero antilatrodectus, Provincia de Buenos Aires. 1979-1988.

\begin{tabular}{lcc}
\hline Sintomas & Ne & $\%$ \\
\hline $\begin{array}{l}\text { Sin sintomas } \\
\begin{array}{l}\text { Solamente } \\
\text { locales }\end{array}\end{array}$ & 7 & 2,5 \\
$\begin{array}{l}\text { Solamente } \\
\text { generales }\end{array}$ & 137 & 7,1 \\
$\begin{array}{l}\text { Locales y } \\
\text { generales }\end{array}$ & 116 & 48,8 \\
$\begin{array}{l}\text { Sin } \\
\text { información }\end{array}$ & 1 & 41,3 \\
\hline Total & 281 casos & $100 \%$ \\
\hline
\end{tabular}

De acuerdo con diversos autores ${ }^{6,8,10,11} \mathrm{y}$ a presente experiencia, el veneno de Latrodectus no provoca signologia local como ampollas, edema oscuro, necorsis cutánea y/o equimosis. Si, dolor en el sitio de la picadura, acompañado com mialgias, transpiración fria $e$ hipertensión. De la Tabla puede inferirse que sólo el $48,8 \%$ de los casos corresponderia estrictamente a accidentes por Latrodectus. Los casos que presentaron sintomas locales y generales, como asimismo los asintomáticos, deberian considerarse como incorrecta transmisión de la sintomatologia en el llenado de la FE. Sin embargo, en esta evaluación fueron incluidos todos ellos, debido a que todos habian recibido tratamiento con suero antilatrodectus.

Con referencia al tiempo transcurrido desde el accidente hasta la aplicación del suero, el $46 \%$ se realizó entre las 3 primeras horas, el $24 \%$ de la cuarta a la sexta hora, llegando el $5 \%$ a más de $24 \mathrm{~h}$. El pico en las 3 primeras horas, se debe a que en este periodo de tiempo, la manifestación de los sintomas de latrodectismo ya es muy evidente y sumamente dolorosa, haciendo que el accidentado recurra rapidamente al auxilio médico. La demora en el resto de los casos, podria deberse a um error en la información anamnésica, salvo en aquellos casos donde se duda que se trate de un verdadero latrodectismo.

En los Centros Antiponzoñosos con mayor experiencia en estos accidentes, las indicaciones terapéuticas con referencia a la dosis aplicada fueron respetadas en la mayoria de los casos. En cambio en los Centros donde el latrodectismo no es frecuente, el médico actuante indicó una dosis más (total $4 \mathrm{ml}$ ). El porcentaje de reacciones alérgicas, posteriores a la aplicación del suero, fue del $5 \%$, correspondiendo de estos el $47 \%$ a reacción inmediata y el resto a reacción tardia (enfermedad del suero) con manifestaciones entre $\operatorname{los} 7$ y 10 dias de la aplicación.

El $100 \%$ de las personas tratadas con el suero antilatrodectus, manifestó disminución de la sintomatologia una hora después de su aplicación, y una casi total recuperación del accidentado dentro de las tres horas.

Con referencia a la possibilidad de captura del animal, los datos han demostrado que en sólo el $15 \%$ de los casos es capturada la araña responsable del accidente. El elevado porcentaje de los accidentados que ingesan para su atención sin el animal agresor ( $85 \%$ ) hace que el médico actuante deba diagnosticar en base 
a los sintomas y signos que presenta el paciente.

El pequeño tamaño de la araña, la abundancia con que se encuentra en determinadas zonas, la posibilidad de dispersión aérea y la tendencia a picar ante la presión que se ejerza sobre su cuerpo, por un lado; y por el otro, el tipo de tarea rural del hombre (levantamiento "a mano" de cosechas), la utilización de ropa liviana y suelta, a torso desnudo, y vestimenta apoyada en el mismo ámbito de trabajo, hacen dificil implementar normas profilácticas para evitar los accidentes. Al respecto, Sampayo ${ }^{11}$ cita varios autores que se acuparon del tema experimentando métodos quimicos, fisicos y biológicos, para el control de esta especie, sin obtener resultados satisfactorios. También resultados negativos tuvieron las medidas de prevención que se adoptaron para evitar los accidentes.

Cabe recordar que en la Argentina el latrodectismo se encuentra encuadrado en la Ley de Accidentes de Trabajo (Ley 23.643) y en la Ley de Notificaciones Obligatorias (Ley 15.465), y ante el escaso conocimiento de éstas, se hace necesario difundir más el aspecto médico legal ${ }^{4,5}$.

\section{Conclusiones}

En el periodo estudiado (diez años), en la Provincia de Buenos Aires se registraron anualmente un promedio de 28 casos, determinados como latrodectismo; siendo la mayor frecuencia en los meses de diciembre a marzo; sin ocurrencia de óbito.

El $80 \%$ de los accidentados pertenecian al sexo masculino siendo la gran mayoria de ellos trabajadores rurales.

No se observó predomino de picados en una región corporal en particular.

La mayor parte de los accidentados concurre al médico dentro de las tres primeras horas de producido el accidente.

Las reacciones alérgicas, posteriores al suministro del suero, representaron el 5\% de los tratados.

Puede inferirse que a los efectos prácticos, y para el caso de los accidentes provocados por Latrodectus, el suero antilatrodectus ha resultado eficaz para su tratamiento.

GRISOLIA, C.S. et al. Epidemiologia do latrodectimo na Provincia de Buenos Aires, Argentina. Rev. Saúde
Públ., S. Paulo, 26: 1 - 5 , 1992. São relatados os resultados do latrodectismo e seu tratamento específico durante um periodo de 10 anos (1979-1988), em Buenos Aires, Argentina. São evidenciados dados de distribuição de acidentes por ano, meses, por sexo, residência do acidentado, região corporal da picada, sintomas apresentados, tempo transcorrido entre o acidente $e$ a aplicação do soro antilatrodectus e a captura do animnal agressor. Observou-se um pico entre 1982 e 1983 com 83 acidentes e uma média de 28,1 acidentes anuais, com maior indice de dezembro a março. Com respeito ao sexo e local de residencia do acidentado, $80 \%$ corresponde a homens, sendo principalmente trabalhadores rurais os mais afectados. A maior percentagem dos acidentes localizou-se no antebraço, cintura pélvica e coxa. Com respeito ao tempo transcorrido desde o acidente até a aplicação do soro, $46 \%$ foi realizado entre as três primeiras horas, sendo que foram capturados apenas $15 \%$ dos animais agressores.

Descritores: Aracnidismo, epidemiologia. Latrodectus.

GRISOLIA, C.S. et al. [Epidemiology of latrodectus bites in Buenos Aires Province, Argentina]. Rev. Saúde públ., S. Paulo, 26: 1 - 5 , 1992. Epidemiological data related to Latrodectus bites over a 10-year period (1979-1988) in Buenos Aires Province, Argentina, are presented. Data on distribution of accidents by year, month, sex, residence of person bitten and general symptomatology are given. Statistical analysis showed a mean of 28.1 bites cases per year, $80 \%$ in males, most of whom were farm workers. The Latrodectus bites were localized in arms, pelvic waist and legs. Forty-six per cent of bitten persons visit the clinic within 1 to 3 hours after the incident, and only $15 \%$ capture the animal responsible for the accident.

Keywords: Arachnidism, epidemiology. Latrodectus.

\section{Referências Bibliográficas}

1. ABALOS, J.W. Las arañas del genero Latrodectus de Argentina. Rev. Cent. Museo de La Plata, 4 (1): 29-5I, 1980.

2. ABALOS, J.W. Las arañas del genero Latrodectus en Santiago del Estero. Cordoba, Universidad Nacional de Cordoba, 1986. (Série Ciências Naturales n. 55).

3. ARGentina. Gobierno de La Provincia de Buenos Aires. Leys, etc. Decreto 9307/74 de febrero 25 de 1976. Boletin Oficial (18220): 484, 1976.

4. ARGENTINA. Leys, etc. Ley 15.465 del 25 de febrero de 1976; notificación médica obligatória. Boletin Oficial (19369): 2, 1976.

5. ARGENTINA. Leys, etc. Ley 23.643 del 7 noviembre 1988; accidentes de trabajo. Boletin Oficial (26502): 3, 1988.

6. CERRUTTI, S. Araña del lino: aracnoidismo. Bol. Sanid. Milit. 5: 307, 1910.

7. GONZALEZ, A. Observaciones bioecológicas 
sobre una especie del género Latrodectus de Sierra de la Ventana. Neotrópica, 22 (68): 129-32, 1976.

8. GRISOLIA, C. Araneismo en la Provincia de

Buenos Aires. Buenos Aires, Ministerio de Salud, Direccion Provincial de Medicina Sanitária, 1983. p. 23.

9. MARETIC, Z. \& GONZALEZ-LORENZO, D.

Carácter profesional del Latrodectismo en paises mediterráneos. Rev. Clin. esp., 160 (4): 225-8, 1981.

10. MARTINO, O. Emponzoñamiento humano provocado por venenos de origen animal. Buenos Aires, Ministério de Bienestar Social de la Nación. Subsecretaria de Medicina Sanitaria, 1979.
11. SAMPAYO, R.L. Latrodectus mactans y latrodectismo. Buenos Aires, 1972. [Tesis Doctorado Medicina - Universidad Nacional de Buenos Aires].

12. URTUBEY, N; BAEZ, E; MAGARINOS, E. Epidemiologia del Latrodectismo en Santiago del Estero, Argentina. Rev. Inst. Anim. Venen., 2: 1-28, 1984.

Recebido para publicação em 30/5/90 Reapresentado em 08/10/91 Aprovado para publicação em 29/10/91 